\title{
The First Two Years of Using a Hybrid Learning Approach at a Laptop University
}

\author{
Dr.-Ing. Clemens Martin \\ Assistant Professor \\ Faculty of Business \& Information \\ Technology \\ Faculty of Engineering and Applied Science \\ Clemens.Martin@uoit.ca \\ Tel: 905/721-3111 x 2341
}

Fax: 905/721-3167

\author{
Dr. Marc A. Rosen \\ Professor and Dean \\ Faculty of Engineering and Applied Science \\ Marc.Rosen@uoit.ca \\ Tel: 905/721-8668 \\ Fax: 905/721-3140
}

\section{University of Ontario Institute of Technology 2000 Simcoe Street North \\ Oshawa, Ontario, Canada, L1H 7K4}

\begin{abstract}
This article describes some of the experiences, challenges, lessons learned and success stories during the first two years of using a hybrid learning approach using laptops at the University of Ontario Institute of Technology. We present two different aspects of our experience: the use of technology in and outside of the classroom, and the necessary institutional support for our technology-based learning approach. Both aspects are presented as perceived directly by faculty. In both areas we discuss the approaches that have worked successfully as well as challenges.
\end{abstract}

\section{Introduction}

The University of Ontario Institute of Technology (UOIT), Ontario's newest university, took in its first students in September 2003.

In support of its market driven and technology oriented mandate UOIT decided to require that all offered programs use innovative, technologysupported teaching strategies. As a result of this strategic decision our current students are all equipped with a laptop computer. This laptop computer is loaded with all program-related software as well as selected teaching and learning support tools. The base image for the laptops includes basic office automation components such as a word processor, spreadsheet, desktop database, and a presentation tool alongside with communication tools.
As teaching support tools, all student computers have Silicon Chalk and Secure Exam installed. These client components are complemented with a backend system, such as our Learning Management System, and the Student Portal that ties into the administrative systems for course registration, grade access and administration.

Program-specific software provides students with access to professional grade software systems on their personal laptops and make computer labs superfluous. Our Mechanical Engineering students, for example, are provided with a set of scientific software tools that are used throughout there first- and second-year math and science courses, and engineering packages like SolidWorks and Unigraphics for Computer Aided Design tasks.

In this paper we discuss the pros and cons of using a web-based Learning Management System as it pertains to education in engineering and information technology. We describe approaches to introduce inclass interactive elements that help encourage wider participation in large classes as well as innovative online learning objects in programming education.

We present early results of an ongoing Teaching Innovation Project aimed at supporting the instructor through the identification and localization of students in the classroom.

We discuss the advantages of using the computer as a testing and examination tool and some lessons learned, as well as remaining issues. The technology allows for many communication paths between students in the classroom and outside of it, which are not yet 
sufficiently controllable. We present our initial attempts to use Silicon Chalk's Class Management facilities and its limitations.

On the IT support side, we demonstrate the processes that we use at UOIT to standardize the technology environment and to ensure a common software infrastructure for our students.

We describe the challenges in managing the IT infrastructure and the approaches that UOIT took to bridge the gap between the academic units and our IT services department.

We will conclude with an outlook on our future plans on using technology-supported education.

\section{Learning Management System}

\subsection{Communication $\mathrm{Hub}$}

The University of Ontario Institute of Technology uses WebCT as its Learning Management System [1]. WebCT serves as a Learning Portal for all students and contains common communications functions such as Mail, Discussion Forums, Chat Rooms, as well as private and public calendars.

In addition to these generic functions, there is an online component available for each UOIT course.

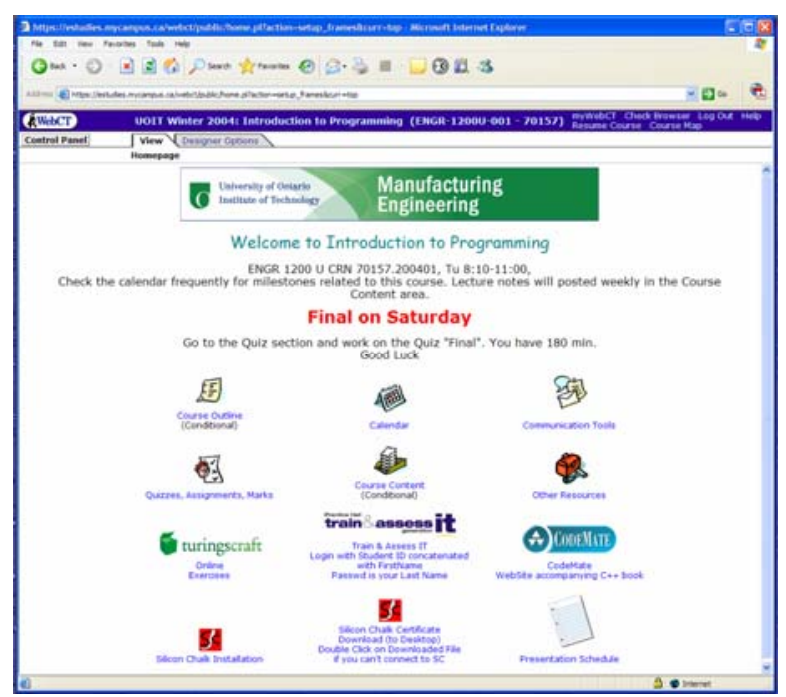

Figure 1: Course Homepage in the Learning Management System

These course specific modules contain handouts, reading lists, presentation slides, course notes, as well as modules to publicize and submit assignments and on-line quizzes (see next section).

Many of these tools can be used without a formalized Learning Management System and some more specialized tools provide better functionality than the combined set of tools within the LMS. The embedded web-based mailing system is somewhat more cumbersome to handle than a specialized email client, while grade calculation is handled more smoothly in a spreadsheet than with the LMS functions.

The main advantage of using one LMS for all tasks lies in its consistency. The University's Innovation Centre has developed a set of templates, in order to harmonize the look and feel of the courses. These templates can be modified by each instructor (Figure 1). Students know where to find their assignments, where they have to turn to communicate with their instructors, or where to look for important announcements.

The common calendar in which all important events from all courses are entered is an invaluable tool for organizing time. Academic standing in all courses is visible to the students through one single tool.

One of the more serious drawbacks in our current implementation is the usage of multiple mail accounts for students. They get mail access for LMS specific mail through WebCT, and instructors encourage students to use only this communication path for oneto-one communication.

Students also get an Internet connected mail-account through our portal, but they prefer to use other free mail services for their out of class communication. A significant percentage admits to not checking this account regularly for mail.

Because the WebCT mail account, which students check for new mail several times per day, is not reachable from the outside and the portal mail account is not read regularly, it is not easy to reach a student by e-mail from other parts of the organization - such as a student advisor, Dean's or Registrar's office which don't have access to the courses in the LMS.

We will have to merge the two different mailing worlds in the future to simplify this communication path between our students and the university.

\subsection{Online Testing}

The Learning Management System supports a web based testing environment that can be used for in and out of class student assessments.

A number of different questions types such as yes/no, multiple choice, fill-in-the-blanks, calculated responses as well as textual responses are supported. Except for the long-text responses all of the above can be automatically graded by the system including the assignment of part marks for multiple choice and fillin-the-blanks.

Questions can be arranged in question sets from which the LMS can generate randomly ordered subsets, and the sequence of multiple choice answers can be randomized. Quizzes can be set to have limits on the 
available work time as well as on the time period in which students can take them.

We started out using the testing environment for out of class lab-style assignments, in which students can choose when they want to take the quiz. This typically worked well.

Moving to an in-class usage for ad-hoc quizzes, midterms and finals initially posed some performance problems in the back-end systems in a network environment that had not completely stabilized. Together with our IT services department we evaluated the performance limitations and decided on investment in additional equipment to make the inclass testing work even with our larger classes of up to 250 students.

The technology has matured enough in the last year so that we can use in-class testing quite extensively. There are still some remaining pitfalls: in each large class there are students whose laptop brakes down just before the test, or is infected by a virus, or is misconfigured by the student. Power supplies and network cables are frequently missing items. Popup blockers, as they are included in many Internet Browsers addons, pose problems when the quizzing environment, which uses a pop-up window, is started. We have adopted a strategy of providing students with an information handout on what to do and what not do, and run a "warm-up" quiz in the last lecture preceding an important exam. This irons out most of the technology problems on the student side. A few spare network cables, power supplies, and - for important exams - a couple of spare laptops in addition to welltrained invigilators, who know how to turn off the pop-up blockers, will take care of most of the remaining problems. In cases in which all quick fixes don't get the student going, a paper copy of the exam is available to ensure the student gets to write the exam. According to our experience in the past year, all these problems combined amount to less then $5 \%$ of a class with a tendency to fewer problems towards the latter half of the academic year and fewer problems with second year students then with first years.

\section{Tools Enhancing Interactivity in the Classroom}

\subsection{Silicon Chalk Basics}

One of the challenges in teaching large classes is the lack of student responsiveness to faculty questions. This reticence can be attributed to a number of reasons. Students might be reluctant to speak up because they feel intimidated when among large numbers of peers, are just generally shy regarding speaking up in public, or don't feel comfortable speaking English because it is not their native language.

We have started adopting Silicon Chalk [2] (SC) to help students participate in in-class activities without the fear of being noticed by their classmates. Using a computer-based interactive tool also caters to a generation of students who have grown up with Internet based technology and are more comfortable 'speaking-up' when they can use technology.

Silicon Chalk provides a set of tools with which interactivity can be supported. SC is mainly a software that supports in-class usage, where it is run on faculty and student laptops during lectures. When students join an activity - such as a lecture - a connection between the instructor's machine and the connected student machine is established and information can be exchanged.

In its most basic usage model, any activity on the instructor's laptop is sent to the connected students along with a recording of the instructor's voice. On the student's machine this broadcast is recorded by the student's installation of Silicon Chalk. While this recording takes place, the student can annotate the presentations in class. This recording can be replayed later and additional notes can be added.

This mode is easy to adopt for every faculty member and does not interfere with a typical instruction style. Students have commented on some limitations on the usefulness of recordings in discussion-oriented classes, because student contributions are not picked up very well in the recording with the standard wireless microphones that UOIT currently uses. In fall 2005 a pilot with omni-directional microphones will be run.

A full semester of recordings that use presentations and instructor audio fits on a single CD.

\subsection{Adding Interactivity with Silicon Chalk}

We have begun using the interactive tools of Silicon Chalk in the past semester. SC provides tools with which quick polls can be performed and results of these polls can be discussed in class. In addition the instructor can identify students who voted in favour of a particular answer and then have student defend their opposing points of view. Using this approach, discussions can be somewhat more easily started in an otherwise passive classroom.

The interactive tools can also be combined with the use of other technology in the classroom. An example is given in Figure 2. Here an in-class exercise consists of a file that is sent out as instructions through Silicon Chalk's Sharebox and students reply with a response file. The same methodology can also be supported through general e-mail or the Learning Management 
System, but the advantage of using the Sharebox lies more in convenience of usage.

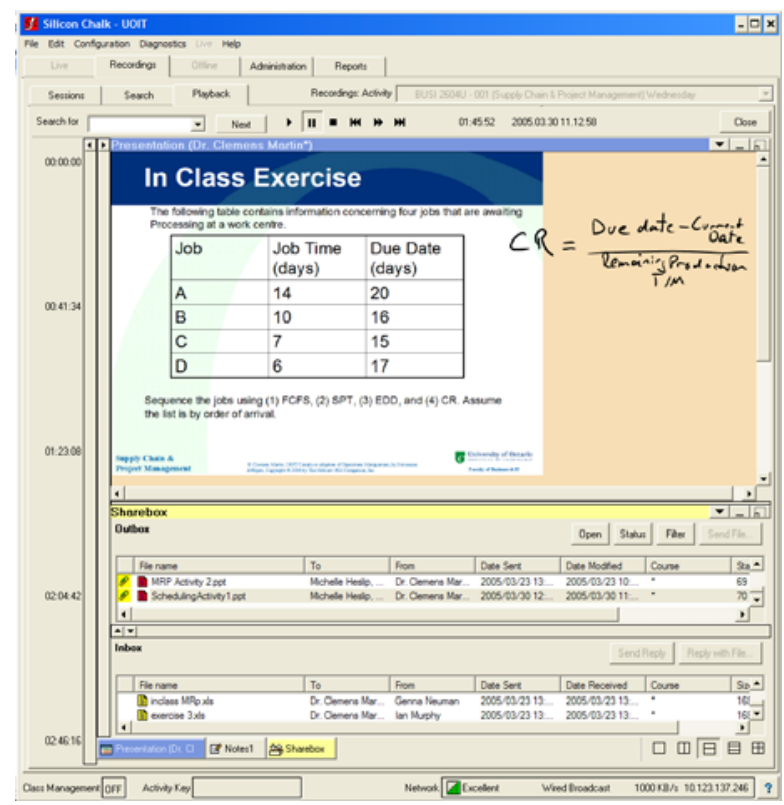

Figure 2: Interactive Elements with Silicon
Chalk

The instructor sees the replies to the sent file in the Sharebox Inbox, and can click on the incoming file to display it on the projection screen to discuss certain aspects of the student's reply.

\subsection{Controlling the Classroom}

While absenteeism and not paying attention is an old problem in university education, technology adds a dimension to this problem. Putting a laptop in the hands of students - particularly in the early semesters can result in easily accessible distraction from lectures and class activities.

Silicon Chalk has built in tools with which the instructor can control which applications can be executed on a student machine. In a programming class the list of allowed application might be limited to Silicon Chalk and the Software Development Environment. The instructor for a statistics class may limit the list to a spreadsheet application and a statistics software package (Figure 3).

The inherent danger in using this feature in class is that students who don't want to participate just won't join the Silicon Chalk in-class activity. We have found that giving out some percentage of the course mark for participation in Silicon Chalk activities results in drastically higher participation rates.

We have used the classroom control feature in exam situations and to limit the allowed applications to the exam-relevant ones and explicitly prohibit any communication programs. The major drawback of this approach is that access to an Internet browser has to be allowed so that students can access the Web based testing system. This opens the door for all web based communication tools such as web-mailers or web based instant messaging systems.

This potential communication path needs to be closed by additional network based controls. We have had some success with the usage of SecureExam [4] for exam situations that don't require the student to access anything but the quizzing environment and a text processor.

We are currently investigating how we can allow the usage of any application while still maintaining control over the communication that takes place.

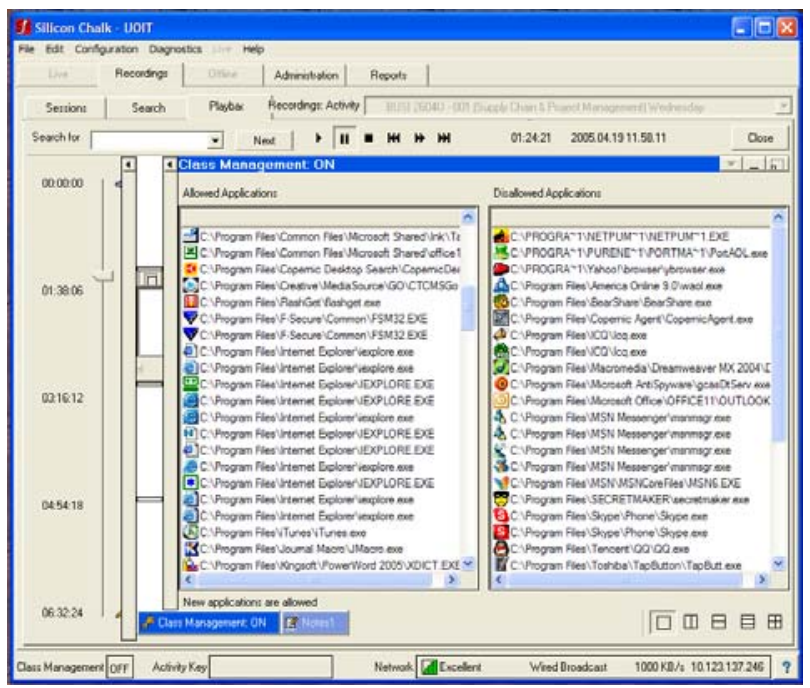

Figure 3: Allowed and Disallowed Application in the Classroom Management Tool

\section{Who's There? A Teaching Innovation Fund Project}

UOIT's student satisfaction surveys indicate that students have a better learning experience when their professors can address them by their names. While some colleagues are gifted enough to learn their student names, even for larger classes with 250 students, most find themselves at a limit between 40 and 60 students.

The University currently funds a project through its Teaching Innovation fund that is intended to help instructors with identifying the students who attend their classes. The project uses information from the mobile networked learning environment to identify which students (or, better, which student laptops) are in a classroom and where these laptops are located. 
With this information a $3-\mathrm{D}$ view of the classroom is dynamically generated and presented to the instructor through a web based interface (Figure 4). A picture of the student is shown together with the student name at the location where the laptop is located by the software, and the instructor can address this student by name. For larger classrooms and lecture halls, a set of pre-defined view points allows the instructor to zoom into zones in the room to identify students who are not recognizable on the overview.

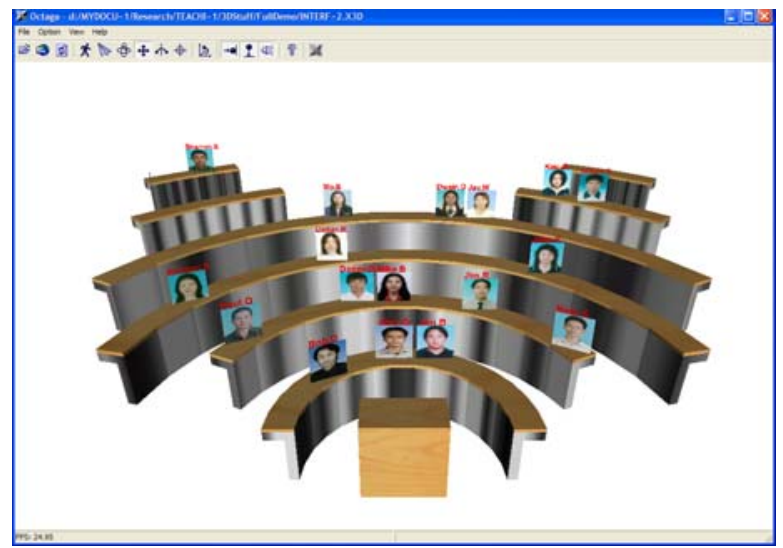

Figure 4: User Interface Prototype of the 'Who's There?' Project

In addition the software can be used for identification purposes in exam settings and to take attendance.

The technology behind the scenes makes use of the ITinfrastructure of the university. All classrooms are wired and students plug in their laptops when they come to class. The information on which laptop is plugged into which port in the classroom is read from the network switches and matched up via the laptop's MAC address, and the student's picture and name. The information on which port on the switch is associated with which outlet in the classroom is held in an underlying database and the location of this port is used to place the picture into the classroom image.

In a second phase of the project a location service based on the wireless signal of the laptops will be added to the software.

\section{Innovative Learning Objects}

One example of enhancing the learning experience for the student with IT comes from an Introduction to Programming class that all engineering students take in first year.

Students who learn a programming language, particularly those doing so for the first time, struggle with two problems simultaneously: Are they using a correct syntax and does their program what they want it to do?

Syntax errors cause compiler error messages that are hard to understand for first timers, and many students believe that they are 'done', once they fix all syntax errors.

We are using a set of online-exercises [3] to address this problem. Students receive a subscription to an external online-service that is covered through their mobile learning fee. They get access to over 200 online exercises that accompany an introductory course using Java, $\mathrm{C}$, or $\mathrm{C}++$ as a programming language.

The main advantage of these exercises is that the feedback goes beyond the typical compiler error message. The service interprets the answer and points the student in the right direction to fix the error - on the syntactical and on the semantic side. In the presented example the answer contains a logical error and the response of the online tool points out where this error might be (see Figures 5 and 6).

The exercises can be used as self study tools but also be bundled as assignments and combined with submission deadlines.

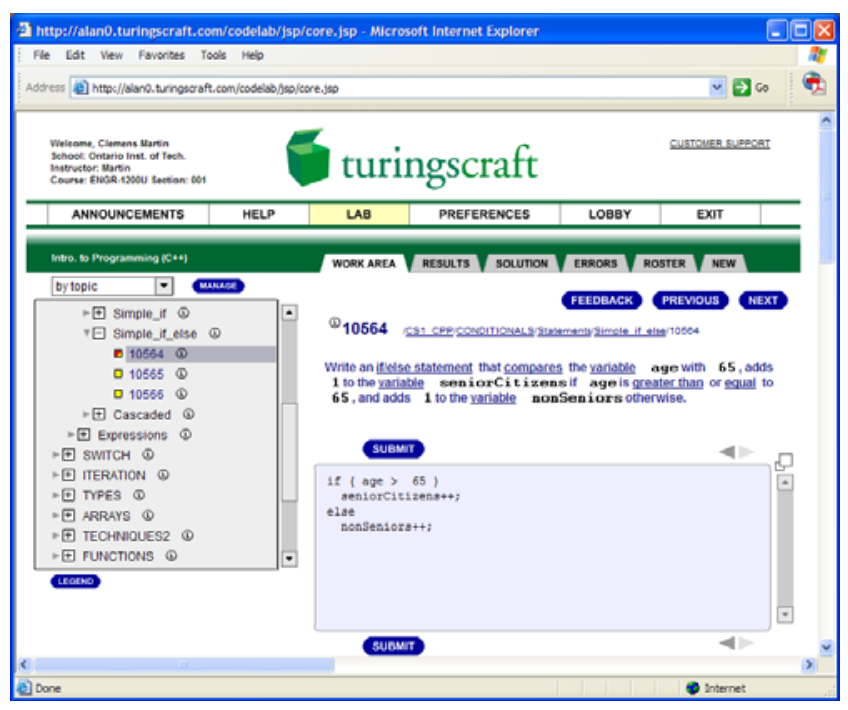

Figure 5: Programming Exercises with Turingscraft

On the instructor side it is possible to look at detailed evaluations on common mistakes that can be used to address recognized problems in in-class settings or in a class accompanying discussion forum. 


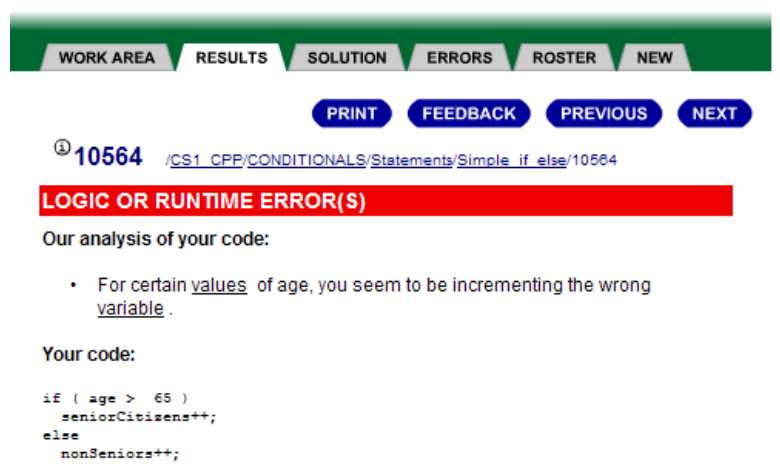

Figure 6: Enhanced Feedback on Incorrect Answers

\section{Enabling Processes}

UOIT relies heavily on its IT infrastructure and on the functionality of the laptops in the students' hand. One of the important success factors therefore is the service around this technology. Our network infrastructure including access to the Internet - has to be running without interruptions. Services need to be in place to repair or replace a student computer within a minimal amount of time.

Standardization of the computer model to a stable product that is supported by the vendor over a longer period of time is mandatory.

We have established and are refining processes between faculties and the IT service organization to submit requirements for academic needs early enough to allow for testing, procurement, integration and imaging. It has been a learning curve for the academic and the services side to recognize the mutual needs and operative constraints, but we are constantly improving.

In the initial years the definition of laptop images sets of programs for cohorts of students such as a first year engineering student - was still possible. The challenge of identifying these images will increase with the growing number of differentiations in the existing programs, new programs and students who are studying "out of phase". We will therefore adopt a course-based imaging model in which the software that is loaded on the student's machine depends on the courses in which the student enrols.

We also will have to address the proliferation of software programs that essentially perform the same task to keep the cost of the mobile program manageable. Currently faculty have the discretion to choose a software package for a course and consolidation of these requirements takes place only to a limited amount - particularly when more than one Faculty is involved in teaching a program. This lack of coordination can result in the installation of a set of software packages that perform an almost identical task.

Addressing this problem is one of the tasks for the coming years.

\section{Outlook}

UOIT will continue to evaluate and implement the usage of technology in the university classroom. As tablet technology becomes more affordable we will pilot its usage by students.

In some areas we will also introduce more hybridized course offerings in which we replace parts of the traditional lecture with other face-to-face components, e.g. in the form of team coaching of project work.

Security in the exam setting remains an important topic and might be a logical extension of the described Teaching Innovation Fund project.

Most advances in the use of new methodologies are carried out by one or a few faculty members. It continues to be an institutional task - residing with our Innovation Centre - to organize and formalize the information exchange process, so that the whole University can benefit from the experiences and learned lessons of some individuals.

\section{References}

[1] WebCT Homepage, http://www.webct.com, Last Accessed May 27, 2005

[2] Silicon Chalk, http://www.siliconchalk.com, Last Accessed May 29, 2005

[3] TuringsCraft, http://www.turingscraft.com, Last Accessed May 30, 2005

[4] Secure Exam, http://www.softwaresecure.com/, Last Accessed May 30, 2005 